\title{
PREVALENCE OF METALLO- $\beta$-LACTAMASES PRODUCING Pseudomonas AND Acinetobacter SPECIES IN TERTIARY CARE TEACHING HOSPITAL, GUJARAT
}

\author{
AHIR H.R. ${ }^{*}$, PATEL P.H. ${ }^{1}$, BERRY R.A. ${ }^{1}$, PARMAR R. ${ }^{1}$, SONI S.T. ${ }^{1}$, SHAH P.K. ${ }^{1}$, VEGAD M.M. ${ }^{1}$ AND PATIL S. ${ }^{2}$ \\ 1Department of Microbiology, B.J. Medical College, Ahmedabad-380016, Gujarat, India. \\ 2Department of PSM, Pad. Dr. D.Y. Patil Medical College, Navi Mumbai-400706, MS, India. \\ *Corresponding Author: Email-dr_hitesh84@yahoo.com
}

Received: November 01, 2012; Accepted: November 10, 2012

\begin{abstract}
-
Background \& Objective: Metallo-ß-lactamases (MBLs) are metalloenzymes of Ambler class B and are resistant to clavulanic acid.. They require zinc as co-factor for enzymatic activity and their activity is inhibited by ethylene diamine tetra acetic acid(EDTA) and other metal ion chelating agents. The first plasmid-mediated MBL was reported in Pseudomonas aeruginosa in Japan in 1991. Since then many countries including few reports from India are available regarding the prevalence of MBLs. The present study was conducted to determine the prevalence of MBLs in Pseudomonas spp. and Acinetobacter spp.

Methods: A total number of 2912 clinical isolates of Pseudomonas spp. $(\mathrm{n}=2162)$ and Acinetobacter spp. $(\mathrm{n}=750)$ obtained over a period of one year, were screened for MBL production by CDST \& DDST.

Results: A total of 325 out of 2912 isolates were positive for MBL production; of which 247 (11.42\%) and 78(10.40\%) were for Pseudomonas spp and Acinetobacter spp. respectively.

Conclusion: MBL producing Pseudomonas aeruginosa and Acinetobacter baumannii group are present in this institution, although in low prevalence. However, to prevent the increase in the incidence of these multidrug resistant organisms and to prevent their dissemination, it is important to detect them and make judicious use of antibiotics based on their susceptibility patterns.
\end{abstract}

Keywords- Pseudomonas spp., Acinetobacter spp., Metallo- $\beta$-lactamase (MBL), MBL detection, Combined disk synergy test (CDST), Double disk synergy test (DDST), Imipenem, Ethylenediamine tetra-acetic acid (EDTA)

Citation: Ahir H.R., et al (2012) Prevalence of Metallo- $\beta$-lactamases Producing Pseudomonas and Acinetobacter species in Tertiary Care Teaching Hospital, Gujarat. International Journal of Microbiology Research, ISSN: 0975-5276 \& E-ISSN: 0975-9174, Volume 4, Issue 9, pp.322-325.

Copyright: Copyright@2012 Ahir H.R., et al. This is an open-access article distributed under the terms of the Creative Commons Attribution License, which permits unrestricted use, distribution and reproduction in any medium, provided the original author and source are credited.

\section{Introduction}

The most serious life threatening infections are caused by a group of drug resistant bacteria that have been labeled the ESKAPE pathogens because they effectively escape the effects of antibacterial drugs. According to CDC (Centre for Disease Control) the six ESKAPE bacteria (Enterococcus faecium, S. aureus, K. pneumoniae, Acinetobacter baumannii, Pseudomonas aeruginosa and Enterobacter spp.) cause two third of all hospital acquired infections. Pseudomonas and Acinetobacter have emerged as important nosocomial pathogens. They are widely distributed in nature and their presence in the hospital environment puts debilitated patients, especially those in intensive care units at risk of opportunistic infections by these multidrug resistant pathogens [1]. Pseudomonas aeruginosa is the most commonly encountered multiresistant gram negative pathogen. Acinetobacter baumanii traditionally infects patients in ICUs and burn units but is now being seen in general hospital population and nursing homes. Gram-negative bacteria with acquired metallo- $\beta$-lactamases production have been increasingly reported in some countries, necessitating their detection.

Metallo- $\beta$-lactamases (MBLs) are metalloenzymes of Ambler class $B$ and are resistant to clavulanic acid. They require zinc as cofactor for enzymatic activity and their activity is inhibited by ethylene diamine tetra acetic acid (EDTA) and other metal ion chelating agents. The first plasmid-mediated MBL was reported in Pseudomonas aeruginosa in Japan in 1991 [2].

Pseudomonas spp. and Acinetobacter spp. are the most important nosocomial pathogens with multiple drug resistance (MDR) [3]. Their high prevalence is of great concern because of their intrinsic and acquired resistance mechanisms, limiting the treatment options. Carbapenems are the drugs of choice for penicillin\& cephalosporin resistant Pseudomonas spp. and Acinetobacter spp. infections. However, this scenario is changing with emergence of $\mathrm{MBL}$ 
producing strains.

We determined the prevalence of acquired metallo-beta lactamases (MBL) producing non fermenting Gram negative bacilli in our tertiary care hospital on 2162 Pseudomonas and 750 Acinetobacter isolates.

\section{Materials and Method}

The present study was conducted from July 2011 to June 2012 over a period of one year. A total number of 2162 Pseudomonas and 750 Acinetobacter isolates were isolated from various clinical samples like swab, urine, sputum, pus, pleural fluid, ascitic fluid and blood samples received from indoor patients of hospital including those admitted to intensive care and acute medical care units. The data regarding samples were obtained from the Microbiology Department and the clinical data was obtained from the respective units and wards of the patients.

Samples were cultured on Nutrient agar, MacConkey agar and Blood agar. Confirmation of Pseudomonas and Acinetobacter spp were done by standard biochemical identification tests [4,5]. Kirby Bauer disc diffusion method was used to detect Imipenem resistance and then the isolates were further tested for MBL production by the combined disc synergy test (CDST)[6,7]and the double disc synergy test (DDST)[6,7] The antibiotic susceptibility profile was determined in accordance to CLSI guidelines using Kirby Bauer Disc diffusion method $[8,9]$. Antibiotics tested were gentamicin, amikacin, tobramycin, cefotaxime, ceftazidime, cefepime, ciprofloxacin, levofloxacin, mezlocillin and piperacillin-tazobactam and aztreonam. For Acinteobacter the antibiotics tested were Cefaclor, Cefotaxime, cefepime, ampicillin-Sulbactam, gentamicin, amikacin, moxifloxacin, tetracycline, cotrimoxazole and chloramphenicol.

- The combined-disk synergy test (CDST) was done using the

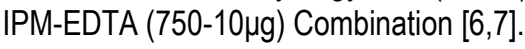

- The Double disk synergy test (DDST) was done using IPM (imipenem)750 $\mu \mathrm{g}$-EDTA $10 \mu \mathrm{g}$ (ethylene diamine tetra acetic acid) $[6,7]$.

\section{Imipenem- EDTA Combined Disc Synergy Test (CDST)}

The Imipenem- EDTA combined disc test (CDST) was preformed as described by Yong et al. The test organisms were inoculated on Mueller Hinton agar as recommended by the CLSI. A 0.5 M EDTA solution was prepared by dissolving $18.61 \mathrm{~g}$. of EDTA in $100 \mathrm{ml}$ of distilled water and adjusting its $\mathrm{PH} 8.0$ by using $\mathrm{NaOH}$. The mixture was sterilized by autoclaving. Two imipenem (10ug) discs were placed on the surface of an agar plate at distance of $25 \mathrm{~mm}$ and 4 ul EDTA solution was added to one of them to obtain a desired concentration of 750ug. The inhibition zones of imipenem and imipenem- EDTA discs were compared after 16 to $18 \mathrm{hrs}$. of incubation in air at $37^{\circ} \mathrm{C}$ [6] In the combined disc test, if the increase in inhibition zone with the imipenem and imipenem- EDTA disc was $\geq 7 \mathrm{~mm}$ than the imipenem alone, it was considered MBL positive [6,7] [Fig-1].

\section{Imipenem - EDTA Double-Disc Synergy Test (DDST)}

The test organisms were inoculated on to plates with Mueller Hinton agar as recommended by the CLSI[8]. An imipenem (10ug) disc was placed $20 \mathrm{~mm}$ center to center from a blank disc contain- ing 4 ul of $0.5 \mathrm{M}$ EDTA (750 ug). Positive results were documented when enhancement of zone of inhibition between imipenem and EDTA disc was $\geq 5 \mathrm{~mm}[7]$.

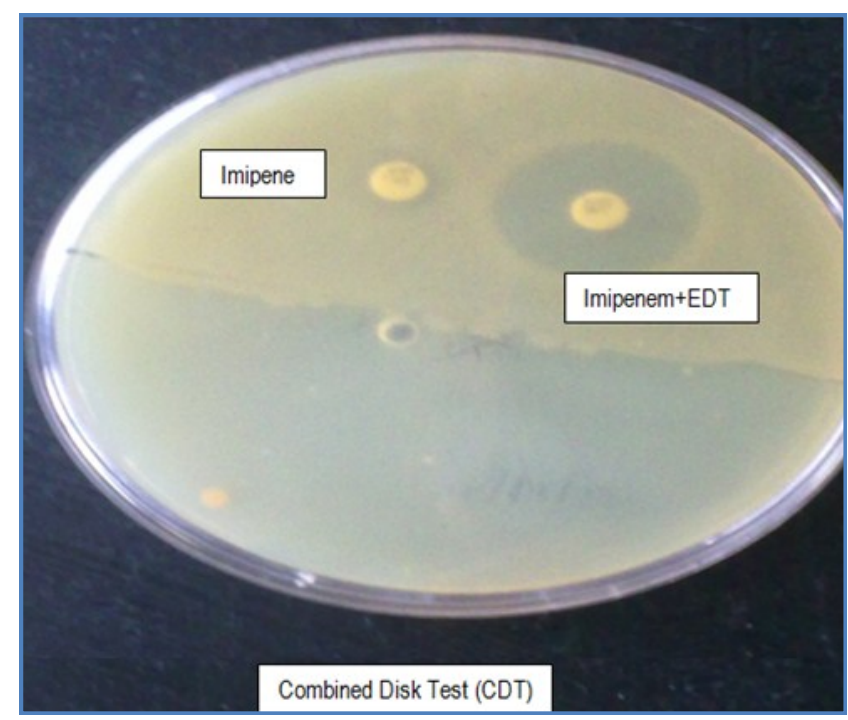

Fig. 1- Combined Disc Synergy Test showing enhancement of zone diameter of $>7 \mathrm{~mm}$ around Imipenem-EDTA disc

\section{Result}

A total of 2162 Pseudomonas spp. isolates and 750 of Acinetobacter spp. were tested for particular antibiotic sensitivity patterns against a panel of antibiotics. Out of them multidrug resistant including imipenem resistant isolates were tested by CDST and DDST $[6,7]$.

In our study maximum number of MBL positive Pseudomonas spp. and Acinetobacter spp. were isolated from swab samples [Table-1, Fig-2].

Table 1- Sample-wise distribution of MBL

\begin{tabular}{lcc|} 
Sample & Pseudomonas (MBL +VE) & Acinetobacter (MBL+VE) \\
Swab & 90 & 40 \\
Sputum & 15 & 7 \\
Urine & 88 & 8 \\
Pleural Fluid & 28 & 7 \\
Pus & 8 & 5 \\
Blood & 10 & 6 \\
Other Body Fluid & 8 & 5 \\
Total & 247 & 78 \\
\hline
\end{tabular}

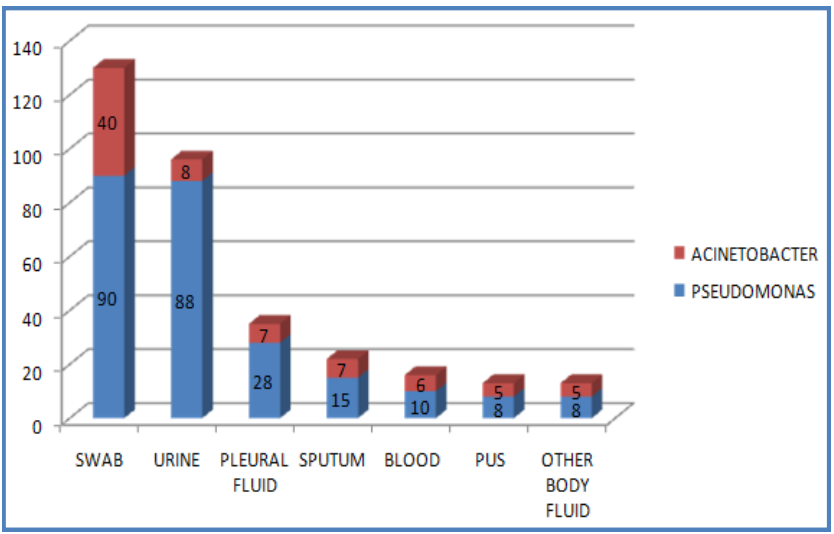

Fig. 2- Sample-wise distribution of MBL 
$247(11.42 \%)$ of pseudomonas and $78(10.40 \%)$ Acinetobacter isolates were confirmed MBL producers phenotypically [Table-2], [Fig3].

Table 2- Prevalence of MBL in Pseudmonas and Acinetobacter spp.

\begin{tabular}{lcc} 
& spp. & \\
Organism & Total & MBL Positive \\
Pseudomonas & 2162 & $247(11.42 \%)$ \\
Acinetobacter & 750 & $78(10.40 \%)$ \\
\hline
\end{tabular}

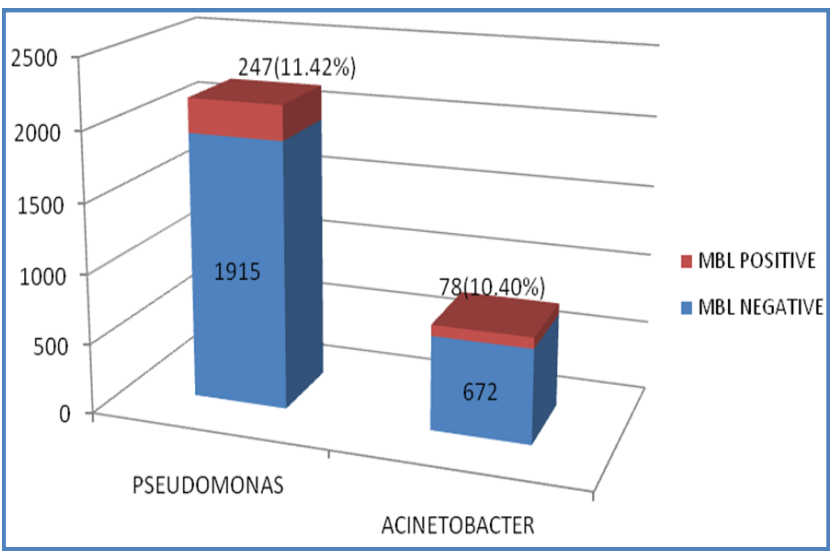

Fig. 3- 2 Prevalence of MBL in Pseudmonas and Acinetobacter spp.

\section{Discussion}

MBL production by Pseudomonas spp. and other gram negative organisms limits the therapeutic options to thetoxic drugs like polymyxin $B$ and colistin.

As MBLs hydrolyze virtually all classes of $\beta$-lactamase, their continued spread will be a clinical catastrophe [10]. With the global increase in the types of MBLs, early detection is crucial [13]. Over the last decade, most of the studies were on different methods of MBL detection in Pseudomonas and Acinetobacter species [11-14]. Though MIC detection remains the gold standard, DDST and CDST are comparable with the former and score well in terms of being simple. easy to perform, reliable and cheap [11-13]. Therefore, these tests can be used in a small laboratory set up also. Other methods for MBL detection used by other workers are: EDTA disc potentiation using ceftazidime, ceftizoxime, cefepime, cefotaxime and the MBL E test $[13,16]$. The high diversity and prevalence of MBL-producing $P$. aeruginosa, Acinetobacter spp., and Enterobacteriaceae isolates have necessitated the search for an accurate MBL screening test. The aim of this study was to evaluate the accuracy of the double-disk synergy test (DDST) and the combined disk (CD) assay to screen for MBL-producing isolates among Pseudomonas spp, Acinetobacter spp isolates that are producers of IMP, GIM, SIM, SPM, or VIM enzymes which ideally should be further confirmed by molecular diagnostic modalities like polymerase chain reaction(PCR). Pseudomonas aeruginosa and Acinetobacter baumannii were common MBL producing isolates in our study [Table-3]. The prevalence of MBLs in Pseudomonas was $11.42 \%$ and in Acinetobacter species it was $10.40 \%$. Other studies have reported the prevalence of $\mathrm{MBL}$ in Pseudomonas as 30.3$36 \%[20,22]$.

Besides being resistant to imipenem, the MBL producers were characteristically resistant to third generation cephalosporins and quinolones[10,15] [Table-4], [Table-5], [Fig-4], [Fig-5] thus limiting the therapeutic options as polymyxin only which too must be used judiciously and not be used as monotherapy [10]. It can be combined with an appropriate aminoglycoside. Aztreonam is the drug of choice for MBL producing Pseudomonas aeruginosa [10]. For treatment of infections caused by multidrug resistant and $\mathrm{MBL}$ producing strains of Acinetobacter species a combination of Imipenem or meropenem along with ampicillin sulbactam remains available [15].

Table 3- Species-wise distribution of MBL

\begin{tabular}{|lclc|}
\hline Pseudomonas spp. & MBL & Acinetobacter spp. & MBL \\
\hline Pseudomonas aeruginosa & 140 & Acinetobacter baumannii & 40 \\
Pseudomonas mendocina & 30 & Acinetobacter Iwoffii & 20 \\
Pseudomonas stutzeri & 25 & Acinetobacter hemolyticus & 10 \\
Pseudomonas putida & 20 & Acinetobacter calcoaceticus & 8 \\
Pseudomonas alkaligens & 32 & - & - \\
Total & 247 & Total & 78 \\
\hline
\end{tabular}

Table 4- Antibiotic resistance pattern in Pseudomonas spp.

\begin{tabular}{lc|} 
Drugs & Pseudomonas spp (N=247) \\
\hline Cefotaxime & $224(90.68 \%)$ \\
Ceftazidime & $180(72.87 \%)$ \\
Cefepime & $190(76.92 \%)$ \\
Piperacillin-Tazobactam & $90(36.43 \%)$ \\
Tobramycin & $195(78.94 \%)$ \\
Amikacin & $120(48.58 \%)$ \\
Ciprofloxacin & $66(26.72 \%)$ \\
Levofloxacin & $34(13.76 \%)$ \\
Mezlocillin & $24(9.71 \%)$ \\
Aztreonam & $12(4.85 \%)$ \\
\hline
\end{tabular}

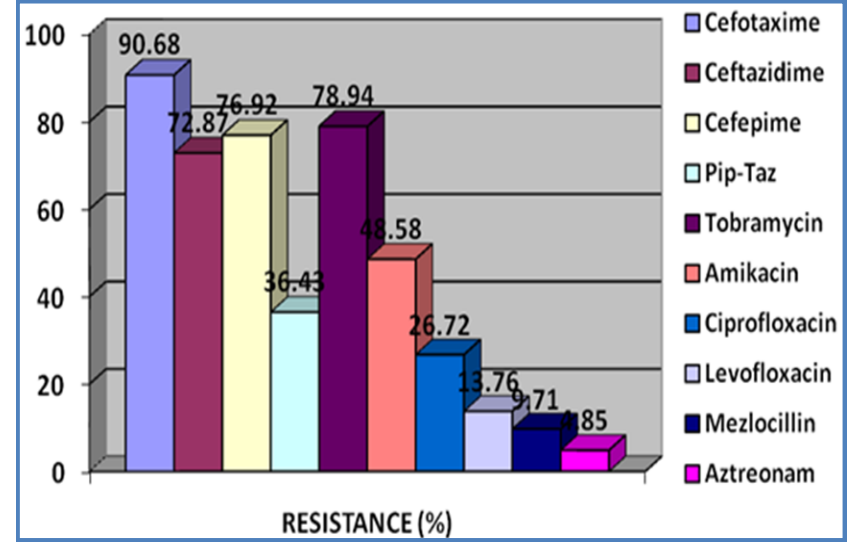

Fig. 4- Antibiotic resistance pattern in Pseudomonas spp.

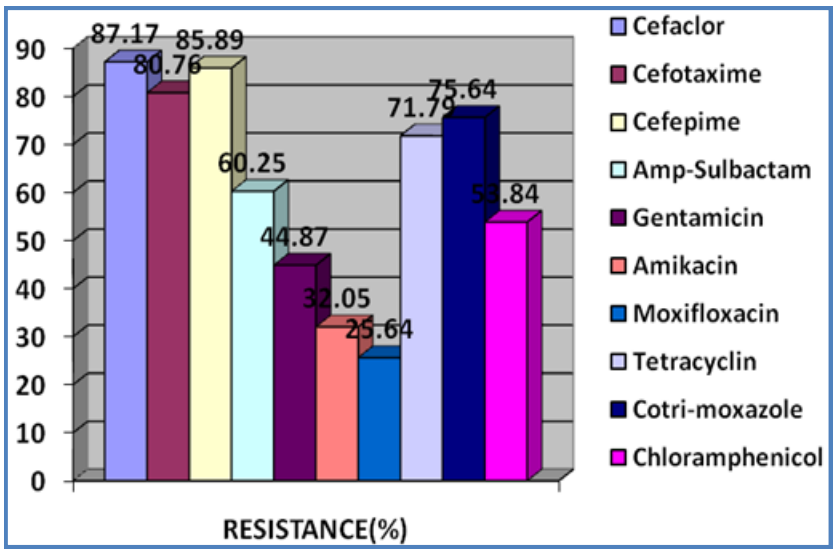

Fig. 5- Antibiotic resistance pattern in Acinetobacter spp. 
Table 5- Antibiotic resistance pattern in Acinetobacter spp.

\begin{tabular}{lc|}
\hline Drugs & Acinetobacter spp (N=78) \\
\hline Cefaclor & $68(87.17 \%)$ \\
Cefotaxime & $63(80.76 \%)$ \\
Cefepime & $67(85.89 \%)$ \\
Ampicillin-sulbactam & $47(60.25 \%)$ \\
Gentamicin & $35(44.87 \%)$ \\
Amikacin & $25(32.05 \%)$ \\
Moxifloxacin & $20(25.64 \%)$ \\
Tetracyclin & $56(71.79 \%)$ \\
Cotri-moxazole & $59(75.64 \%)$ \\
Chloramphenicol & $42(53.84 \%)$ \\
\hline
\end{tabular}

In a Government hospital like ours, that provides health care facilities to the poorest of the poor, the cost constraints evaluation mandate the prescription of a drug if the drug is not available in the hospital formulary. Patient affordability is another factor which has to be kept in mind before the drug is prescribed.

\section{Conclusion}

MBL producing Pseudomonas aeruginosa and Acinetobacter baumannii group are found in wards, although in low prevalence. However, to keep them in check, regular detection of this bacteria and judicious use of antibiotics to which they are still susceptible is mandatory. Increased prevalence of carbapenem resistance being acquired by MBL is particularly reported for Pseudomonas(11.42\%) and for Acinetobacter(10.40\%). Rapid detection of MBL producing gram negative bacteria is necessary for therapy and to prevent their further dissemination. A high prevalence of MBL among Pseudomonas aeruginosa is a critical problem representing a practical therapeutic challenge. Emergence of $\mathrm{MBL}$ producing P.aeruginosa and Acinetobacter species in ICUs is alarming and reflects excessive use of carbapenems. In ICUs the selection pressure is the greatest which selects multi drug resistant strains over the competitive flora [17] Therefore a strict antibiotic policy should be followed in intensive care areas to prevent further spread of MBLs. Clinicians should prescribe antibiotics judiciously. Timely implementation of strict infection control practices and antibiotic resistance surveillance programs should be carried out from time to time [13]. Detection of MBLs by either CDST or DDST should be routinely performed in all microbiology laboratories for all imipenem-resistant isolates, which will help to reduce morbidity and mortality in these patients. Though it is desirable to detect MBL producers at the earliest by routine laboratory testing, one must exercise care while interpreting phenotypic results based on inhibitor synergy. PCR should be done to validate such results.

\section{References}

[1] Sarkar B., Biswas D., Prasad R. (2006) Indian J. Pathol. Microbiol., 49, 44-8.

[2] Butt T., Usman M., Ahmed R.N., Saif I. (2005) J. Pak. Med. Assoc., 5, 302-4.

[3] Stephane Corvec, Nathalie Caroff, Eric Espaze, Cecile Giraudeau, Henri Drugeon, Alain Reynaud (2003) Journal of Antimicrobial Chemotherapy, 52, 629-35.

[4] Collee, et al., Mackie \& MaCartney Practical Medical Microbiology., 120-384.

[5] Koneman E.W., et al., Color Atlas and Textbook of Diagnostic Microbiology, 5th edition. 69-8.
[6] Yong D., Lee K., Yum J.H., Shin H.B., Rossolini G.M., Chong Y. (2002) J. Clin. Microbiol., 40, 3798-801.

[7] Lee K., Lim Y.S., Yong D., Yum J.H., Chong Y. (2003) J. Clin. Microbiol., 41, 4623-9.

[8] Clinical Laboratory Standards Institute (2007) Seventeenth Informational Supplement., M100-S17, 27, 38-41.

[9] Bauer A.W., Kirby W.M., Sherns J.C., Turck M. (1966) Am. J. Clin. Pathol., 45, 493-6.

[10]Walsh T.R., Toleman M.A., Poirel L., Nordmann P. (2005) Clin. Microbiol. Rev., 18, 306.

[11]Pitout J.D.D., Gregson D.B., Poirel L., McClure J.A., Le P., Church D.L. (2005) Clin. J. Microbiol., 43, 3129-35.

[12]Marra A.R., Pereira C.A., Gales A.C., Menezes L.C., Cal R.G. de Souza J.M., et al. (2006) Antimicrob Agents Chemother., 50, 388-90.

[13]Behera B., Mathur P., Das A., Kapil A., Sharma V. (2008) Indian J. Med. Microbiol., 26, 233-7.

[14]Jayakumar S., Appalaraju B. (2007) Indian J. Patho. Microbiol. 50, 922-5.

[15]Perez F., Hujer A.M., Hujer K.M., Decker B.K., Rather P.N., Bonomo R.A. (2007) Antimicrob. Agents Chemother., 51, 347184.

[16]Walsh T.R., Bolmstrom A., Qwarnstrom A., Gales A. (2002) J. Clin. Microbiol., 40, 2755-9.

[17]Shanthi M., Sekar U. (2009) J. Assoc. Phys. India., 57, 636-45. 